\title{
Phase space holes and elementary radiation events
}

\author{
R. Pottelette ${ }^{1}$ and J. Pickett ${ }^{2}$ \\ ${ }^{1}$ CETP-CNRS, 4 avenue de Neptune, 94107 Saint-Maur des Fossés, France \\ ${ }^{2}$ Department of Physics and Astronomy, The University of Iowa, Iowa City, IA, USA
}

Received: 21 May 2007 - Revised: 4 October 2007 - Accepted: 21 October 2007 - Published: 26 November 2007

\begin{abstract}
Using high-time resolution measurements from the FAST and CLUSTER spacecraft we analyze different types of fine frequency structures of Auroral Kilometric Radiation (AKR). Based on the results from previous numerical simulations, we emphasize that the recorded frequency structures are the ones which are expected to occur on the low- and high-potential sides of an accelerating double layer. Electron holes producing steep perpendicular velocity gradients on the horseshoe velocity distribution function appear to be responsible for generating AKR fine-frequency structures. However, both ion and electron hole dynamics seem to play a major role in the generation of the subtle, fine structures moving across the radiation spectrum.
\end{abstract}

\section{Introduction}

In the auroral upward current region, the concerted action of the parallel acceleration of the electrons and the mirror effect of the magnetic field lead to the formation of an incomplete ring or horseshoe distribution. Under these circumstances, the energetic electron distribution $F_{e}$ still remains peaked at $0^{\circ}$ but has large $\partial F_{e} / \partial v_{\perp}>0$ over a wide range of parallel velocities, where $v_{\perp}$ is the component of the electron velocity perpendicular to the magnetic field. FAST satellite observations have now provided conclusive evidence that the electron-cyclotron maser driven by such an unstable horseshoe distribution is responsible for the coherent generation of AKR (Ergun et al., 2000)

High spectral resolution of the AKR emission strongly suggests that a large part of the emission is made up of narrowband drifting structures which are believed to be real elementary emitters (Gurnett and Anderson, 1981). The simplest assumption is thus to identify the emission sources with

Correspondence to: R. Pottelette

(raymond.pottelette@cetp.ipsl.fr) such real, drifting small-scale physical objects. It was recently underlined that nonlinear phase-space holes play a key role in generating different AKR frequency structures (Pottelette et al., 2001, 2003; Pottelette and Treumann, 2005; Mutel et al., 2006). Since the scale size of electron and ion holes is a few Debye lengths, the observed narrow bandwidth $(\sim 100 \mathrm{~Hz})$ of AKR fine structures is easily accounted for. In the AKR source region the Debye length is $\sim 100 \mathrm{~m}$, so that typical sizes for electron holes range from $100 \mathrm{~m}$ to $1 \mathrm{~km}$. In order to be capable of emitting, these objects must be embedded in an unstable particle distribution and must be able to stimulate radiation. This is possible when realizing that the electron holes are effectively positive charges on the electron background, which implies that they will interact in a certain way with the main electron component. This interaction consists of the electron holes becoming attracted by the bulk of the electrons and thus trapped in the electron horseshoe distribution while at the same time growing in amplitude as their absolute depth will be conserved. This can lead to a collection of holes in a more narrow space region which then acts as the radiation source (Pottelette et al., 2001).

In this paper we provide experimental evidence that the presence of a localized magnetic field-aligned potential drop not only favours the growth of the electron-cyclotron maser instability but in addition leads to the generation of finefrequency structures in the AKR spectrum. Actually, this potential generates a large number of electron holes via the two-stream instability and ion holes via the current-driven ion acoustic instability which manifest themselves as subtle, fine structures moving across the radiation spectrum.

\section{Electrostatic double layers}

It has long been recognized that a class of electrostatic structures generally referred to as double layers can support a potential jump confined to a narrow spatial region in a

Published by Copernicus Publications on behalf of the European Geosciences Union and the American Geophysical Union. 

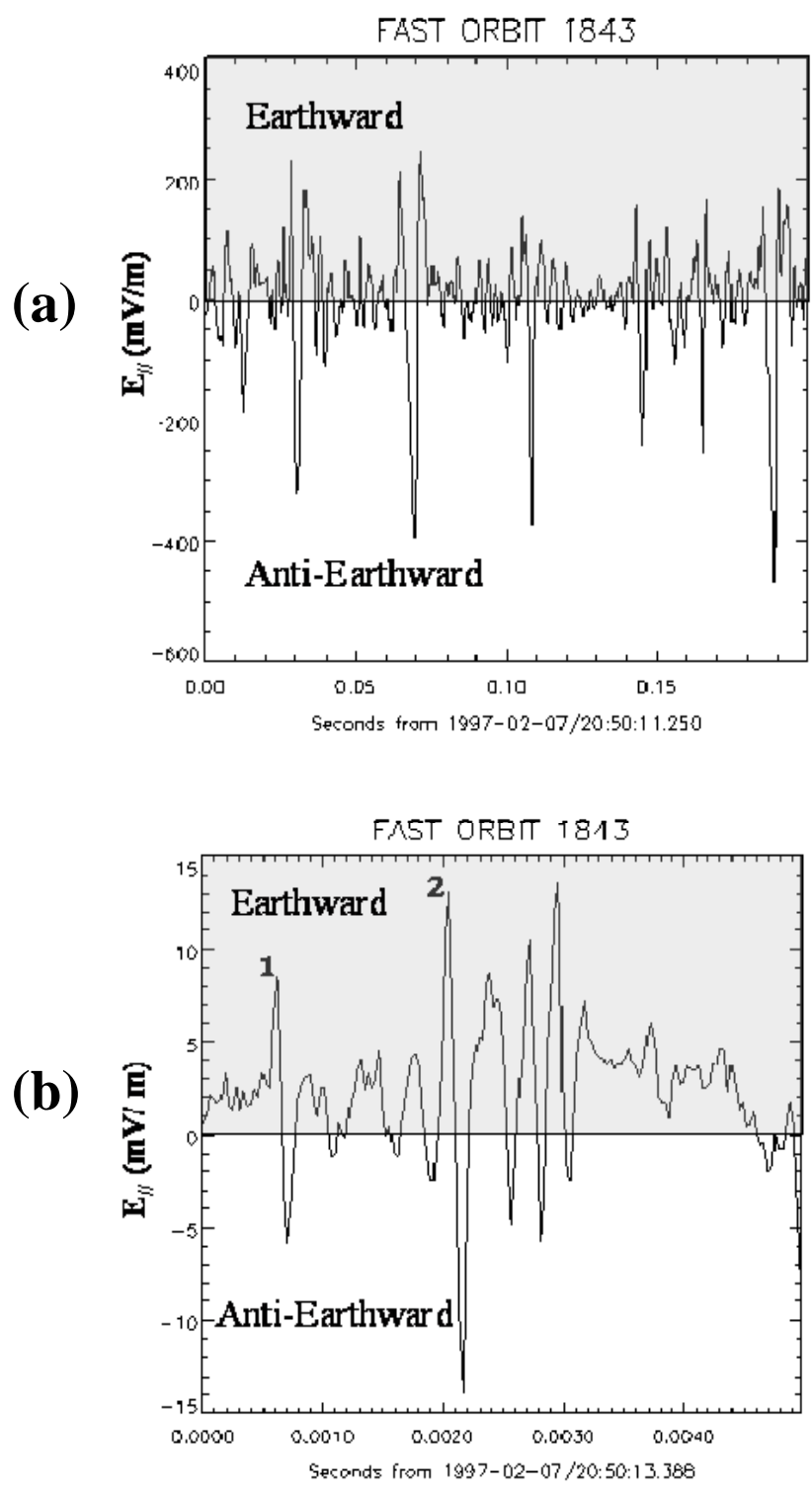

Fig. 1. High time resolution of quasi-parallel electric field VLF waveform measured by FAST in the upward current region when it was located in the vicinity of a double layer: (a) Low-potential side showing a large amplitude chain of equally spaced tripolar electric field structures (b) High-potential side showing well defined bipolar electric field structures.

current-carrying collisionless plasma (Block, 1971). The approximate electric potential drop across such an electrostatic double layer (also called "electrostatic shock") in collisionless plasmas can be easily estimated from the energy conservation law (Tidman and Krall, 1971; Jasperse et al., 2006):

$$
\left|j_{/ /} E_{/ /}\right| \approx d\left(n k_{B} T_{e}\right) / d t
$$

Here $j_{/ /}$is the parallel current density, $E_{/ /}$the parallel electric field, and $n k_{B} T_{e}$ the thermal energy density of the background plasma. Assuming that the shock is quasi-stationary and causes a density depression $\Delta n$ by evacuating the plasma in a region of length $\Delta x$ while moving at speed $v_{s}$, the potential drop $\Delta \Phi=E_{/ /} \Delta x$ can be approximated by:

$\Delta \Phi \approx \frac{\Delta n}{n} \frac{n}{n_{b}} \frac{v_{s}}{v_{b}}\left(\frac{k_{B} T_{e}}{e}\right)$

where $n_{b}$ and $v_{b}$ are the density and the drift velocity, respectively, of the electron beam which sustains the parallel current. Typical shock velocities are of the order of the ion acoustic speed. Hence for $\mathrm{keV}$ beam electrons, the velocity ratio is of the order $<10^{-2}$. Assuming a nearly complete evacuation of the plasma $\Delta n \sim n$ as it is frequently observed in the upward auroral current region where AKR is generated, the total potential difference is $\Delta \Phi<10^{-2}\left(n / n_{b}\right) \mathrm{kV}$. Since the electron beam-to-plasma density ratio is $\sim 10^{-2}$ $10^{-3}$ the potential difference can reach several $\mathrm{kV}$, implying that a few of these strong double layers suffice to account for the necessary potential drops in the aurora. Alternately, if the density depression is much less, many very weak double layers are required to account for the potential drop. These weak double layers will be asymmetric, soliton-like structures or phase space holes.

\section{Turbulence generated in the neighbourhood of a double-layer}

Double layers once immersed in the plasma will necessarily accelerate particles along the magnetic field, thereby generating locally strong turbulent processes leading to the formation of phase-space holes. The ion holes are the result of a two-stream instability (Gray et al., 1990; Main et al., 2006), while the electron holes are generated by an electron-electron instability. The physics of the formation of these holes is highly non-linear and cannot be accessed by a perturbation approach. The only way of investigating their evolution is through numerical simulations which have been performed by several authors (Newman et al., 2001, 2002; Singh, 2000; Goldman et al., 1999, 2003). These simulations have produced important results: in particular they have shown the asymmetric character of the turbulence generated in the regions located upstream (low-potential side) or downstream (high-potential side) of a double layer.

The asymmetry is well-illustrated by Fig. 2 of Goldman et al. (2003). The space-time plot of the time history shows that initially, on the high-potential side of the double layer, many electron holes are generated by an electron-electron instability, and are moving earthward at a speed comparable to the accelerated electrons. These holes are small amplitude and have a bipolar, parallel electric-field signature.

At a later time, on the low-potential side, the double layer releases ion holes which move anti-earthward with a speed comparable to the accelerated ion beam. These ion holes are themselves ramps on the electron scale, i.e., they reflect 
low energy electrons thereby leading to the generation of secondary electron holes which remain captured between the ion holes and the double layer ramp for a relatively long time. A chain of electron and ion holes with a tripolar electric-field signature begins to move anti-earthward with a speed comparable to the ion-beam drift velocity.

It should be stressed that the nonlinear structures predicted by the results of this numerical simulation have definitely been observed in the heart of the acceleration region (Pottelette and Treumann, 2005) in the vicinity of a double layer associated with localized anti-earthward directed parallel fields. As illustrated in the $200 \mathrm{~ms}$ time sequence of Fig. 1a, tripolar electric field profiles are observed on the low-potential side of this double layer. These electric field structures reflect the presence of a chain of electron and ion holes. As expected, they have very large amplitudes reaching $\sim 800 \mathrm{mV} / \mathrm{m}$ peak-to-peak with typical time duration of approximately $5 \mathrm{~ms}$. The anti-earthward (negative) field in their centres is flanked by two weaker-field earthward (positive) peaks, in agreement with the tripolar structures moving upward. In contrast, on the high-potential side of the same double layer, the $5 \mathrm{~ms}$ time sequence shown in Fig. $1 \mathrm{~b}$ reveals the presence of well defined bipolar electric field structures (labelled 1 and 2) which reflect the presence of electron holes. As expected, the holes in this chain have much smaller amplitudes than those moving in the low-potential side. Their typical amplitude is of the order of $15-30 \mathrm{mV} / \mathrm{m}$ peak-to-peak and their time duration is $\sim 200 \mu \mathrm{s}$; their polarity is in agreement with a downward motion. The experimental results unambiguously reveal the presence of electron holes in the upward current region which so far has been overlooked.

The numerical simulations also show that, on the lowpotential side, after having interfered with other newly created electron and ion holes and having reached large enough amplitudes, the electron holes belonging to this chain attain enough momentum to break through the initial double layer ramp and escape earthward. The large-amplitude electron holes quasi-periodically interrupt the double layer until it has again reformed.

These observations, associated with the theoretical predictions, prove that all of the turbulent dynamics are basically confined to the low potential side of the double layer where a dynamical coupling between electron and ion holes takes place. Moreover, it appears that the phase-space holes generated by a double layer interact with this layer and can even disrupt it. However, the numerical simulations performed so far have neglected the formation of the horseshoe distribution which, in the presence of a magnetic-field-aligned electric field, is unavoidable. Therefore, these simulations do not contain any signature of radiation.

\section{Radiation from nonlinear phase space holes}

As previously emphasized, the emission of AKR is not continuous but consists of the superposition of elementary radiation events, the emitted radiation of which superimposes to generate the apparently continuous AKR spectrum. In light of the previous discussion, it seems quite natural to assume that the elementary radiators are the electron holes which are generated in the plasma and move on the background of the horseshoe distribution.

Numerical simulations show that electron holes are situated in the plateaued initial electron beam (Singh, 2003). In order to contribute to the generation of radiation via the electron-cyclotron maser mechanism, an electron hole must be able to modify the original horseshoe-distribution function by imposing a steep perpendicular-velocity-gradient on this electron-distribution function. Since the density of an electron hole is conserved, its amplitude grows when moving into the denser part of the electron distribution (Pottelette et al., 2001). In the region of the hole, this implies a local decrease in electron density and, at the same time, an increase in velocity space gradients. Therefore, the gradients on the electron distribution function increase steeply in both directions - parallel and perpendicular to the magnetic field when the hole deforms and enters the horseshoe distribution. In this way, deformed electron holes produce steep, perpendicular gradients $\partial F_{e} / \partial v_{\perp}>0$ in the horseshoe velocity distribution at the hole boundaries and act as efficient radiation emitters.

As illustrated in Fig. 30 of Treumann (2006), emission occurs at the edge of the electron hole where $\partial F_{e} / \partial v_{\perp}>0$, while at the edge where $\partial F_{e} / \partial v_{\perp}<0$, the hole absorbs radiation. The emissions and absorptions take place at slightly different frequencies due to the finite width $\Delta$ of a hole in velocity space. The frequency gap $\delta f$ between emission and absorption can be easily estimated from the value of the resonant velocity $v_{r}$ required by the maser mechanism at the location of the hole in velocity space where it forms a dip. The resonance condition leading to a resonant interaction between the electromagnetic waves and energetic electrons occurs at the Doppler shifted electron-cyclotron frequency (Eq. 1 of Treumann, 2006). For strictly perpendicular propagation, as observed in the AKR source region, the resonant velocity is such that:

$v_{r}^{2}=2 c^{2}\left(1-\frac{f}{f_{c e}}\right)$

where $f_{c e}$ is the electron-cyclotron frequency. Differentiating Eq. (3), one easily gets:

$\frac{\delta f}{f_{c e}} \sim \frac{\Delta}{c} \frac{v_{r}}{c}$

Assuming $10^{-2}<\Delta / v_{r}<10^{-1}$ as derived from numerical simulations, the AKR spacing between emission and absorption should be such that $10^{-4}<\delta f / f<10^{-3}$. This 


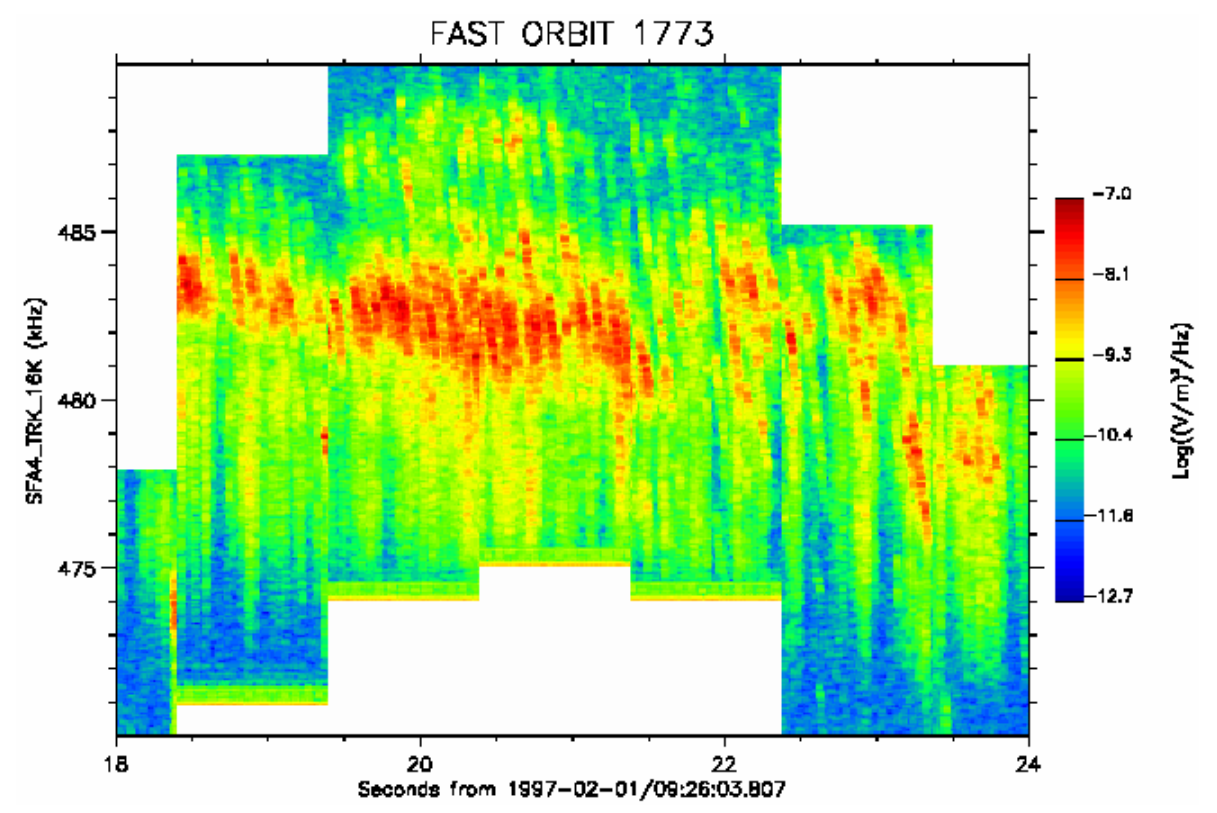

Fig. 2. Frequency-time spectra of striated AKR bursts recorded on the FAST spacecraft when it was located in the nightside magnetosphere at an invariant latitude of $68^{\circ}$.

corresponds to a frequency gap on the order of $\sim 100 \mathrm{~Hz}$. Consequently, signatures of narrow emission and absorption lines in the AKR spectrum should indicate strong evidence for the identification of electron-hole radiation.

In short, the main striking results derived from numerical simulations which can be applied to the understanding of the generation of AKR fine-frequency structures are the following:

1. The large number of small-amplitude electron holes escaping at high speed from the high-potential side of a double layer and moving earthward should form the elementary background radiation sources;

2. The occurrence of intense, fine-structured emission bands (known as striated AKR) can be explained only when ion holes and secondary electron holes are produced on the low-potential side of a double layer. These electron holes are trapped between the ion holes and move anti-earthward at the ion-drift velocity.

3. A small number of large-amplitude electron holes are trapped on the low-potential side and move along with the double layer. They are reflected until they reach sufficient amplitudes to break through the double-layer potential. These trapped holes produce intense "inverted U" type narrow-band signatures in the AKR spectrum (Pottelette and Treumann, 2001).

\section{Observations}

In order to illustrate the different types of AKR fine frequency structures, we use high time resolution data provided by the FAST and CLUSTER spacecraft that track AKR in different altitude ranges.

Assuming dipole magnetic field geometry, by transforming the induced frequency drifts into spatial radial velocities $V$, one gets

$V \approx \frac{R_{E}}{3}\left(\frac{f_{c o}}{f}\right)^{1 / 3} \frac{1}{f} \frac{d f}{d t}$

where $f_{c o}$ is the electron cyclotron frequency at the surface of the Earth $\left(f_{c o} \sim 1.7 \mathrm{MHz}\right), f$ the observed frequency, and $R_{E}$ is the Earth radius (Gurnett and Anderson, 1981).

As the AKR emissions take place near the electron cyclotron frequency $f_{c e}$, assuming again a dipole magnetic field geometry, a bandwidth $\Delta f$ corresponds to a radial size $\Delta z$ of the elementary radiation structures such that

$\Delta z \approx \frac{R_{E}}{3} \frac{\Delta f}{f}\left(\frac{f_{c o}}{f}\right)^{1 / 3}$

At FAST satellite altitudes where $f \sim 400 \mathrm{kHz}$, one gets $\Delta z=0.85 \mathrm{~km}$ for $\Delta f=100 \mathrm{~Hz}$, and $V=8 \mathrm{~km} / \mathrm{s}$ for $d f / d t=1 \mathrm{kHz} / \mathrm{s}$.

\subsection{Striated AKR emission}

Figure 2 shows an example of a specific type of AKR fine structure known as striated AKR (Menietti et al., 2000). These $6 \mathrm{~s}$ of data were acquired by the tracker experiment on 


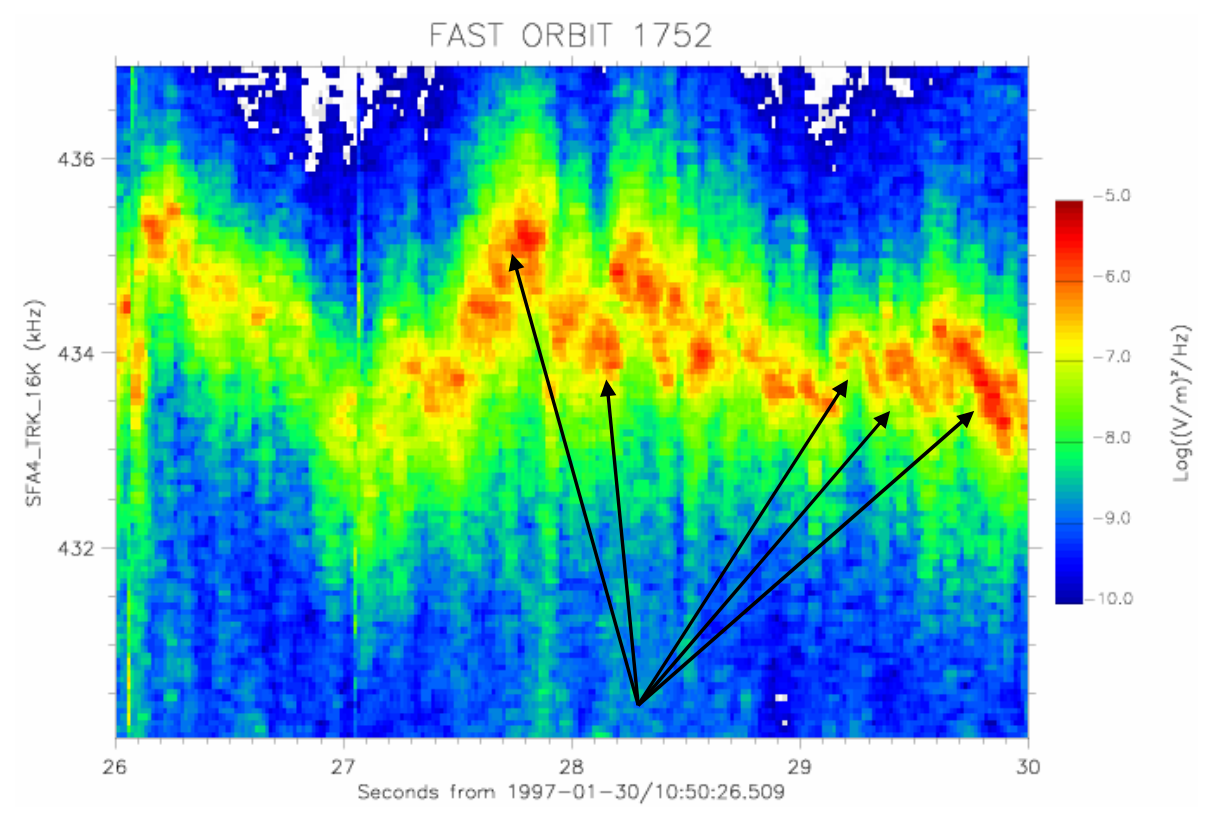

Fig. 3. "Inverted U" type AKR frequency structures recorded by the FAST spacecraft at about 22:00 MLT and at invariant latitude of 69.
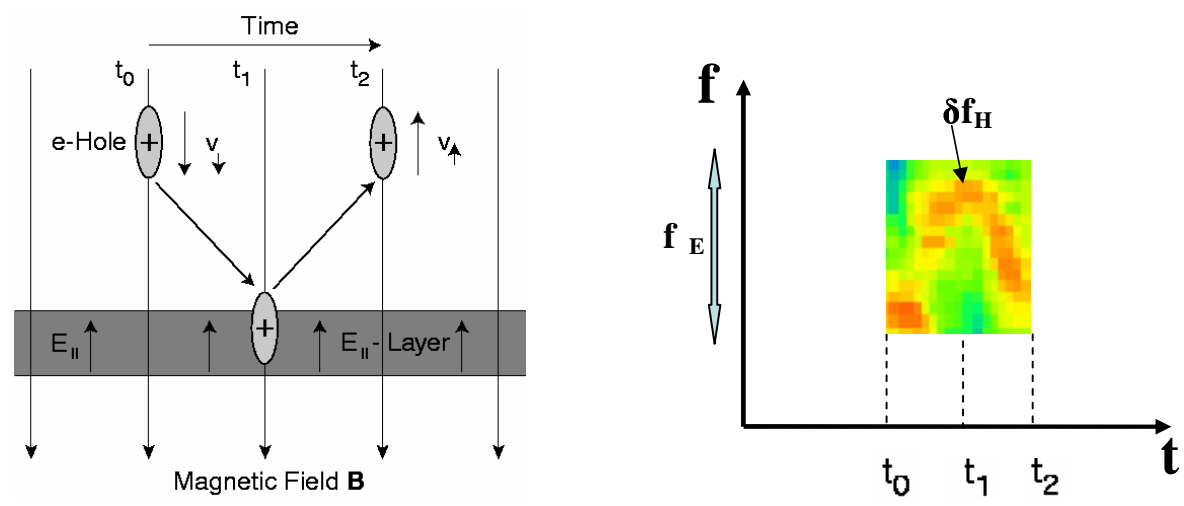

Fig. 4. (Left) Reflection of an electron hole at a parallel electric-field layer. The hole moving earthward and being a positive charge is rejected by the anti-earthward directed field-aligned electric field. (Right) "Inverted U" type frequency structure radiated by the hole when rejected by the electric-field layer.

board the FAST spacecraft when it was located in the nightside magnetosphere at an invariant latitude of $68^{\circ}$. In this figure we see a large number of negative sloped traces in the frequency range from 485 to $475 \mathrm{kHz}$, corresponding to local electron cyclotron frequencies at altitudes of $\sim 3500 \mathrm{~km}$. The striated AKR fine structures are those which are expected to be generated on the low-potential side of a double layer in the plasma regime where ion and electron holes move together anti-earthward at about the ion-drift velocity. As previously illustrated in Fig. 1a), trains of nested ion and electron holes are characterized by large-amplitude; isolated parallel electric-field structures of tripolar polarity (Pottelette and Treumann, 2005). The elementary radiation sources observed in Fig. 2 move towards lower frequency across the
AKR spectrogram in agreement with the hypothesis that they are generated by slow electron holes moving together with ion holes in an anti-earthward direction along the magnetic field lines.

The moving structures have an average $d f / d t \sim-30 \mathrm{kHz} / \mathrm{s}$ and their instantaneous bandwidth is $\sim 100 \mathrm{~Hz}$ which is about the frequency resolution of the experiment. Using Eqs. (5) and (6) leads to a speed $V \sim 200 \mathrm{~km} / \mathrm{s}$ and to a size $\Delta z \sim 0.65 \mathrm{~km}$ along the magnetic field lines for the elementary radiators. The velocity range corresponds to ion drift speeds (Bounds et al., 1999) and such a small source size matches with a few Debye lengths. These structures last typically $0.2 \mathrm{~s}$, corresponding to several thousands of plasma periods in the AKR source region where $f_{p} \sim 5 \mathrm{kHz}$. Such time 


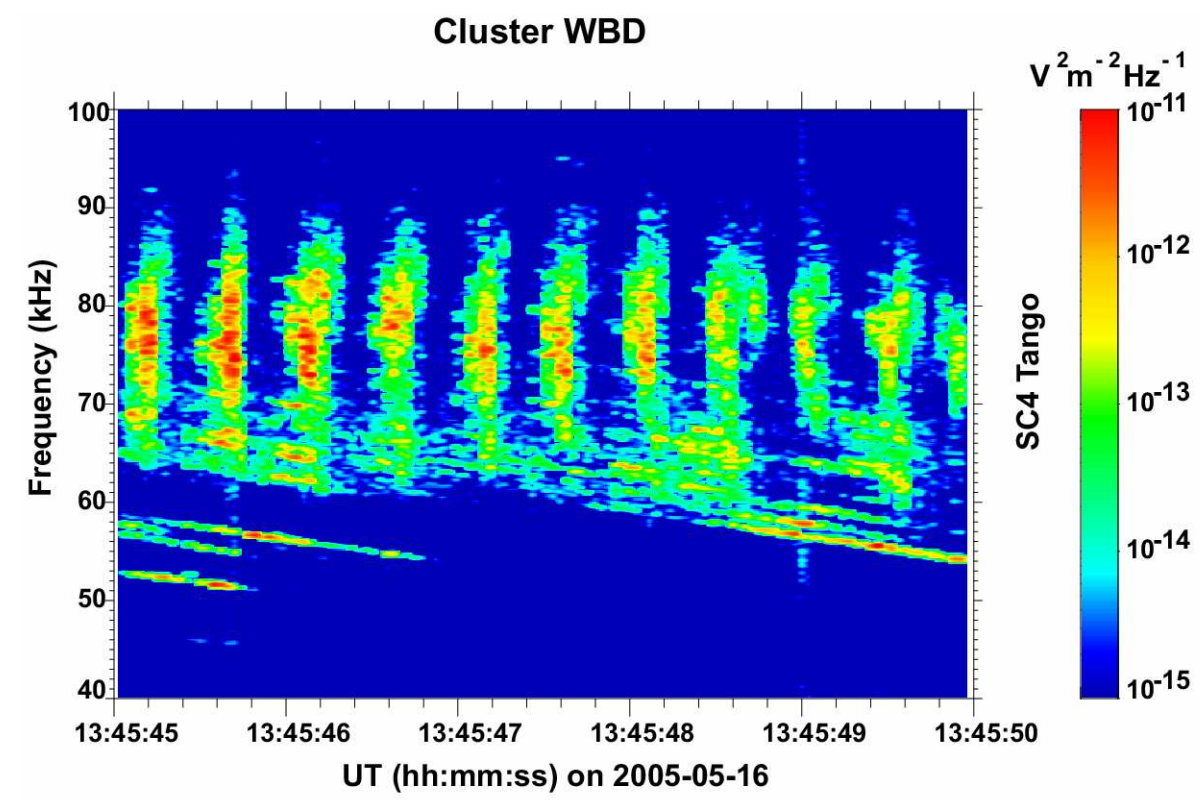

Fig. 5. Spectrogram showing pulsed and striated AKR observed on a Cluster satellite located in the nightside magnetosphere at $14.2 R_{E}$ and invariant latitude of $80^{\circ}$ on 16 May 2005 over a $5 \mathrm{~s}$ interval.

duration is in very good agreement with the lifetimes of electron holes predicted by numerical simulations. During this elapsed time the elementary radiation sources travel about $40 \mathrm{~km}$ inducing perturbations in a $\sim 5 \mathrm{kHz}$ frequency range as observed.

\section{2 "Inverted U" AKR fine structures}

So far, "inverted U" type narrow-band signatures in the AKR spectrum have only been reported from the high-time resolution data acquired by the FAST spacecraft (Pottelette et al., 2001; Treumann, 2006). They are illustrated in Fig. 3, where $4 \mathrm{~s}$ of data taken by the FAST tracker are displayed. The arrows present in this figure show well-defined drifting elementary emission sources approaching a reflector from above (increasing frequency), being reflected when hitting the reflector and returning upward (decreasing frequency) along the magnetic field lines. The radiation pattern of these sources forms a kind of inverted $\mathrm{U}$.

It is tempting to identify the reflector with a parallel electric-field layer as schematized in Fig. 4. Being a positive charge, the electron hole is rejected by the presence of a field-aligned electric field. An underestimated order of magnitude of the vertical size of the layer can be approximated from the overall frequency width $\Delta f_{E}$ of the induced perturbations (see Fig. 4, right). A systematic analysis reveals that $\Delta f_{E} \sim 1 \mathrm{kHz}$ corresponding to $\Delta z_{E} \sim 8 \mathrm{~km}$.

It must be stressed that just as in the case of striated AKR, the lifetime of the elementary radiating structures is $\sim 0.2 \mathrm{~s}$, and their instantaneous bandwidth $f_{H} \sim 100 \mathrm{~Hz}$. As in the former case, these sources can be safely identified as elec- tron holes in phase space which are of the spatial size of just a few Debye lengths. In the present case, the radiation is caused by large-amplitude electron holes trapped on the lowpotential side of the double layer. They are reflected until they reach sufficient amplitudes to break through the doublelayer potential (Newman et al., 2001; Goldman et al., 2003).

\subsection{Pulsing AKR emissions}

Figure 5 displays pulsing AKR emissions recorded in the nightside magnetosphere at $14.2 R_{E}$ and invariant latitude $80^{\circ}$ by the WBD (wide band data) high-time resolution experiment on board the Cluster mission (Gurnett et al., 1997). This event took place during the recovery phase of a large storm in a $10000-13000 \mathrm{~km}$ altitude range given the observed frequency range of the radiation. The AKR amplitude appears to be modulated at frequencies near $2.8 \mathrm{~Hz}$ typical for Pc1-pulsations (R. Schreiber, personal communication). In addition to this modulation, the spectrum of AKR radiation reveals two different types of fine-frequency structures. The first one, known as striated AKR, is associated with moderate negative-slope frequency drifts and is recorded below a quasi-steady frequency $f^{*} \sim 70 \mathrm{kHz}$ at the low frequency edge of the spectrum. The second type is associated with an abrupt frequency expansion into higher frequencies above $f^{*}$.

These observations can be interpreted as the result of a local acceleration by a pulsing parallel electric field layer located at a quasi-steady altitude of $\sim 11000 \mathrm{~km}$ for which the value of the electron cyclotron frequency is $f^{*}$. Upstream of this layer, at frequencies smaller than $f^{*}$, the usual striated 
AKR emissions are recorded. The mean slope of these AKR drifting emissions is $-2.4 \mathrm{kHz} / \mathrm{s}$, corresponding to a trigger speed of $210 \mathrm{~km} / \mathrm{s}$ using Eq. (5), which matches with antiearthward propagating ion hole speeds. Downstream from the accelerating layer, electron holes escaping earthward at high velocity are responsible for the abrupt frequency expansion of the AKR emissions above $f^{*}$. What is noteworthy in the latter emissions is the presence of a well-defined series of closely spaced absorption and emission bands (separated by a few hundreds Hertz) which are characteristic of AKR generation by electron holes. Similar structuring, but somewhat less defined, can also be observed in Figs. 2 and 3.

\section{Discussion and conclusions}

In order to understand the generation of AKR fine structures one needs first to identify "in situ" which type of turbulence is created by the particle acceleration processes in a given altitude-range of the auroral region. We have emphasized in this paper that different kinds of elementary radiation events have their sources in spatially localized wave structures which are generated in the vicinity of electrostatic double layers (electrostatic shocks).

The phase-space dynamics of electron holes may provide the key to an understanding of the drifting emissions obtained from horseshoe velocity distributions when electron holes are generated in the current-carrying plasma. We have indeed identified the sources as electron holes in phase-space which are of the spatial size of just a few Debye lengths. The presence of such elementary radiators logically explains the narrow bandwidths, sometimes of the order of $\Delta f / f \sim 10^{-4}$ to $10^{-3}$ which are recorded (Yoon and Weatherwax, 1998). In addition, the quasi-systematic signatures of narrow emission and absorption lines in the AKR spectrum indicate strong evidence for the identification of electron hole radiation.

Numerical simulations point to a dynamical coupling between ion and electron holes in the presence of a double layer. This coupling is important for the application to the electron cyclotron-maser mechanism. For instance, it gives a natural explanation for the generation of the so-called striated AKR emissions upstream from a double layer (Pottelette and Treumann, 2005). Alternative suggestions based only on the interaction of an ion hole with a horseshoe electronvelocity distribution (Mutel et al., 2006) are not consistent with the observation of narrow emission and absorption frequency bands in the AKR spectrum as illustrated in Figs. 2 and 5 .

Lastly, it should be emphasized that observations of AKR fine structures provide remote information about the local dynamics of the auroral zone turbulence. In particular, it provides an indication of the existence of layers carrying parallel electric-potential drops. One impressive aspect is the remote sensing of the acceleration processes occurring at high altitudes during the recovery phase of a large storm as shown in
Fig. 5. Fine AKR frequency structures reveal unambiguously the presence of a quasi-steady pulsing parallel electric field located at a fixed altitude around $11000 \mathrm{~km}$.

Acknowledgements. We thank the organizers of the NLW-6 Workshop held in Kyushu, Japan in October 2006 for inviting us to present this work. We also thank the referees for their constructive comments and improving suggestions. The FAST mission is a project of the Space Sciences Laboratory of the University of California at Berkeley run under the auspices of NASA. The authors are indebted to C. W. Carlson and R. E. Ergun for providing the particle and wave data as well as for some useful discussions. This research has been initiated within the France-Berkeley program. Work at the University of Iowa was carried out under NASA GSFC Grants No. NAG5-9974 and No. NNG04GB98G and NSF Grant ATM 03-27450.

Edited by: T. Hada

Reviewed by: P. Yoon and two other anonymous referees

\section{References}

Block, L. P.: Potential double layers in the ionosphere, Cosmic Electrodyn., 2, 184-196, 1971.

Bounds S. R., Pfaff, R. F., Knowlton, S. F., et al.: Solitary Structures Associated with Ion and Electron Beams near 1 Re Altitude, J. Geophys. Res., 104(A12), 28 709-28 717, 1999.

Ergun, R. E., Carlson, C. W., McFadden, J. P., Delory, G. T., Strangeway, R. J., and Pritchett, P. L.: Electron-cyclotron maser driven by charged-particle acceleration from quasi-static magnetic-field-aligned potentials, Astrophys. J., 538, 456-466, 2000.

Goldman, M. V., Oppenheim, M. M., and Newman, D. L.: Nonlinear two-stream instabilities as an explanation for auroral bipolar wave structures, Geophys. Res. Lett., 26, 1821-1824, 1999.

Goldman, M. V., Newman, D. L., and Ergun, R. E.: Phasespace holes due to electron and ion beams accelerated by a currentdriven potential ramp, Nonlin. Processes Geophys., 10, 37-44, 2003,

http://www.nonlin-processes-geophys.net/10/37/2003/.

Gray, P. C., Hudson, M. K., Bergmann, R., and Roth, I.: Simulation study of ion two-stream instability in the auroral acceleration region, Geophys. Res. Lett., 17, 1609-1612, 1990.

Gurnett, D. and Anderson, R.: The Kilometric Radio Emission Spectrum: Relationship to Auroral Acceleration Processes, Physics of Auroral Arc Formation, Geophysical Monograph Series, 25, 341-350, 1981.

Gurnett, D. A., Huff, R. L., and Kirchner, D. L.: The Wide-Band Plasma Wave Investigation, Space Sci. Rev., 79, 195-208, 1997.

Jasperse, J. R., Basu, B., Lund, E. J., and Bourham, M.: Gyrotropic guiding-center fluid theory for turbulent inhomogeneous magnetized plasma, appendix A, Phys. Plasmas, 13, doi:10.1063/12220006, 2006.

Main, D. S., Newmann, D. L., and Ergun, R. E.: Double layers and ion phase-space holes in the auroral upward current region, Phys. Rev. Lett., 97, 185001, 2006.

Menietti, D., Persoon, A., Pickett, J., and Gurnett, D.: Studies of AKR fine Structures Striations Observed by Polar, J. Geophys. Res, 105(A8), 18 857-18 886, 2000. 
Mutel, R. L., Menietti, J. D., Christopher, I. W., Gurnett, D. A., and Cook, J. M.: Striated auroral Kilometric radiation emission: A remote tracer of ion solitary structures, J. Geophys. Res., 111, A10203, doi:10.1029/2006JA011660, 2006.

Newman, D. L., Goldman, M. V., Ergun, R. E., and Mangeney, A.: Formation of Double Layers and Electron Holes in a CurrentDriven Space Plasma, Phys. Rev. Lett., 87, 255001, 2001.

Newman, D. L., Goldman, M. V., and Ergun, R. E.: Evidence for correlated double layers, bipolar structures, and very lowfrequency saucer generation in the auroral ionosphere, Phys. Plasmas, 9, 2337-2343, 2002.

Pottelette, R., Treumann, R. A., and Berthomier, M.: Auroral Plasma Turbulence and the cause of AKR fine structure, J. Geophys. Res., 106(A5), 8465-8476, 2001.

Pottelette, R., Treumann, R., Berthomier, M., and Jasperse, J.: Electrostatic shock properties inferred from AKR fine structure, Nonlin. Processes Geophys., 10, 87-92, 2003, http://www.nonlin-processes-geophys.net/10/87/2003/.
Pottelette, R. and Treumann, R.: Electron holes in the auroral upward current region, Geophys. Res. Lett., 32, L12104, doi:10.1029/2005GL022547, 2005.

Singh, N.: Electron holes as a common feature of double layerdriven plasma waves, Geophys. Res. Lett., 27, 927-930, 2000.

Singh, N.: Space-time evolution of electron-beam-driven electron holes and their effects on the plasma, Nonlin. Processes Geophys., 10, 53-63, 2003, http://www.nonlin-processes-geophys.net/10/53/2003/.

Tidman, D. A. and Krall, N. A.: Shock waves in collisionless plasmas, ChapI, Wiley-Interscience, New-York, 1971.

Treumann, R.: The electron-cyclotron maser for astrophysical application, Astron, Astrophys. Review, 13(4), doi:10.1007/s00159-0060001-y, 2006.

Yoon, P. and Weatherwax, A.: A theory for AKR fine structure, Geophys. Res. Lett., 24, 4461-4464, 1998. 